\title{
Niveau et facteurs de ruptures des premières unions conjugales au Burkina Faso
}

\author{
Bilampoa Gnoumou Thiombiano a et Thomas K. Legrand ${ }^{b}$ \\ alnstitut Supérieur des Sciences de la Population, Université de Ouagadougou, \\ Burkina Faso, E-mail: bgnoumou@issp.bf \\ bDépartement de démographie, Université de Montréal, Canada
}

\section{Résumé}

Nous avons utilisé les données d'enquêtes biographiques individuelle et communautaire pour examiner le niveau et les facteurs de risque de divorce au Burkina Faso. Les résultats révèlent que le divorce y est moins fréquent que dans certains pays d'Afrique subsaharienne, mais le niveau augmente au sein des jeunes générations de mariage. Environ une femme sur dix rompt sa première union au cours de 30 ans de mariage. L'analyse par la régression semi-paramétrique à risque proportionnel de Cox indique que les facteurs de risque de divorce se situent à la fois au niveau individuel et communautaire. Les résultats montrent que les variables liées au cycle de vie familial et matrimonial (infécondité, mariage précoce), à la modernisation (urbanisation, désenclavement du lieu de résidence) et au statut socioéconomique de la femme (autonomie financière) sont des facteurs associés à un plus grand risque de ruptures d'unions. Les facteurs culturels (religion, socialisation, ethnie) introduisent aussi des différences de risque de divorce.

Mots clés: divorce, séparation de couple, femme, Burkina Faso

\begin{abstract}
We used data from two biographical surveys at individual and community levels, to study the level and the risk factors for divorce in Burkina Faso. The results show that divorce is less common than in some subSaharan African countries, but the level is higher among the young generations of marriage. About one in ten women experience divorce during their first marriage. The results from Cox proportional hazards model show that risk factors for divorce are at both individual and community level. Variables related to family lifecycle and marriage (infertility, early marriage), modernization (urbanization, improved accessibility of residences) and socio-economic status of women (financial independence) are more associated with risk factors of separation and divorce. Cultural factors (religion, socialization and ethnicity) also introduce differences in the risk of divorce.
\end{abstract}

Keywords: divorce, separation, woman, Burkina Faso 


\section{Introduction}

Alors que plusieurs travaux ont examiné les facteurs de divorce dans les pays occidentaux, pour l'instant, très peu d'études ont porté sur cette problématique dans les pays d'Afrique subsaharienne. Plusieurs raisons peuvent expliquer cette situation. L'une des raisons est le manque de données appropriées. Les recensements se limitent souvent à collecter l'information sur l'état matrimonial des individus. Les Enquêtes Démographiques et de Santé (EDS) collectent des données sur les dernières unions. Ces données du moment ne permettent pas de reconstituer l'histoire matrimoniale des individus et de suivre le devenir des unions. Pour examiner la dynamique des unions, une approche longitudinale est nécessaire. Mais cette approche longitudinale du devenir des unions a été rarement adoptée en Afrique subsaharienne, notamment en Afrique de l'Ouest, à l'exception de travaux concentrés en milieu urbain (Antoine et Bocquier, 1999 ; Antoine et Dial, 2005 ; Marcoux, 1997, Mouvagha-Sow, 2003) et sur des populations particulières (Hertrich, 1996).

Au Burkina Faso, selon les données de recensements, la proportion de femmes veuves et divorcées est passée de $12 \%$ en 1985 à $10 \%$ (dont $9 \%$ de veuves et $1 \%$ de divorcées) en 2006 . Ces données indiquent un faible niveau des femmes en situation de rupture conjugale. Cependant, la vie hors union étant mal perçue comme dans plusieurs sociétés africaines, en cas de rupture d'union, le remariage intervient souvent rapidement. Aussi, les données transversales ne rendent donc pas compte du niveau des ruptures d'unions conjugales. Néanmoins, selon certains travaux (Lesthaeghe et al., 1989), les ruptures d'union par séparation ou divorce sont fréquentes en Afrique de l'Ouest, et le niveau augmente depuis quelques années (Hertrich et Locoh, 1999; Locoh et Mouvagha-Sow, 2005). Cette instabilité matrimoniale est souvent considérée comme un désir d'émancipation (Thiriat, 1998), voire une source de promotion pour les femmes (Dial, 2007).

Mais globalement, peu d'hypothèses explicatives ont été formulées sur le divorce (White, 1990). Toutefois, quelques hypothèses explicatives ont été formulées dans le cadre général des théories du mariage et de la famille. Une des hypothèses fondée sur la "théorie économique du mariage" attribue l'augmentation de l'instabilité des unions dans les pays occidentaux au cours du dernier siècle à la hausse du travail des femmes (Becker, 1974, 198I ; Becker et al., 1977). Mais cette hypothèse a été peu confirmée par les études empiriques (Mongeau et al., 200I), même si quelques travaux sur la relation travail-famille des femmes l'ont testé (Spitz, 1988 ; Ruggles, 1997a, 1997b; Takyi et Broughton, 2006). Elle a d'ailleurs été très contestée par certains auteurs (Oppenheimer, 1994, 1997a, 1997b). Selon Oppenheimer (1994) le lien entre le travail des femmes et le mariage comprend aussi des effets positifs qui atténuent les effets négatifs. Pour cet auteur, ce sont les difficultés d'insertion économique des hommes qui sont à l'origine de l'augmentation du divorce dans les pays occidentaux. Une autre approche, développée en sociologie de la famille, postule que la stabilité des unions conjugales dépend non seulement des avantages du mariage comme le revenu élevé du mari, mais aussi des obstacles au divorce et des solutions alternatives possibles dont disposent les conjoints. La théorie sociologique du mariage présume que l'opportunité de travail de la femme est une solution alternative au mariage tandis que les pressions sociales et religieuses et les obligations morales, comme la prise en charge des enfants, sont des obstacles au divorce (Levinger, 1965; Udry, 1981).

Cette étude s'inscrit dans l'approche sociologique du mariage et a pour objectif général de contribuer à une meilleure connaissance des ruptures d'unions conjugales par divorce ou séparation de couple au Burkina Faso. Elle a pour objectifs spécifiques d'étudier le niveau du divorce et d'examiner comment certains facteurs individuels et communautaires influent sur le risque de divorce de la première union des femmes. Une attention particulière est accordée aux effets des enfants sur ce risque. Nous supposons par exemple que, dans le contexte du Burkina Faso où la fécondité est très valorisée et essentielle, l'infécondité du couple est un facteur de risque de divorce.

Au Burkina Faso, selon le code des personnes et de la famille (Article 354), le divorce peut résulter du consentement mutuel des époux constaté par un tribunal civil ou d'une décision judiciaire prononçant la dissolution du mariage à la demande de l'un des conjoints (Burkina Faso, 1990). Mais fréquemment, la rupture d'union conjugale se produit par une séparation de fait avec le départ définitif de la femme du foyer conjugal (parfois après plusieurs aller-retours) sans procédure judiciaire, et aucune procédure traditionnelle ne marque le divorce. II est donc parfois difficile de distinguer les couples séparés et ceux divorcés. Ceci étant, dans cette étude, le terme divorce désigne toute rupture d'union autre que le veuvage déclarée par les individus. Dans la section suivante nous présentons une brève revue de la littérature sur le divorce et les hypothèses de 
l'étude. La méthodologie de l'analyse et les résultats de l'étude sont ensuite exposés, avant de terminer par une discussion et une conclusion.

\section{Revue de la littérature et hypothèses}

La majeure partie des travaux empiriques utilisent des données individuelles et le risque de divorce est examiné selon diverses caractéristiques individuelles et parfois du couple. De nombreuses recherches soulignent que le jeune âge au premier mariage est un facteur important de risque de divorce en Afrique et ailleurs (Olusanya, 1970; Booth et Edwards, 1985; White, 1990; Bumpass et al., 1991; Thiriat, 1998; Clarke et Berrington, 1999; Tilson et Larsen, 2000; Antoine et Dial, 2005). Plusieurs hypothèses sont développées pour expliquer cet effet négatif de la précocité du mariage sur la stabilité des unions conjugales. Une des hypothèses est que les individus mariés très jeunes n'ont pas pris suffisamment de temps dans la recherche de leur conjoint (Becker et al., 1977; Thornton et Rodgers, 1987) et qu'ils sont immatures et mal préparés à assumer leurs rôles conjugaux (Amato et Previti, 2003; Booth et Edwards, 1985). En Afrique, selon Locoh (1995), le mariage précoce, généralement arrangé par les familles, est une sorte de "rite de passage" pour la jeune fille, un moyen d'acquérir sa majorité sociale. Ce mariage est souvent vécu comme une contrainte dont la femme essaye de se libérer par le divorce afin d'épouser un homme de son choix.

L'infécondité (ou la sous-fécondité) est également un facteur important de divorce en Afrique (Takyi, 200I). Les familles africaines sont très attachées à l'enfant, la procréation est d'ailleurs la vocation première d'une union, en conséquence, l'infécondité du couple augmente le risque de divorce. Par exemple au Togo, en milieu rural, les unions infécondes ont deux fois plus de risque d'être rompues que les unions fécondes (Thiriat, 1998). Les recherches menées dans d'autres régions ont aussi souligné le risque élevé de divorce des couples sans enfants (Andersson, 1997; Bumpass et al., 1991; South, 1995; Clarke et Berrington, 1999).

Par ailleurs, contrairement à certains pays occidentaux où le faible niveau d'éducation des femmes est associé à un plus grand risque de divorce (Bumpass et al., 1991 ; South, 1995 ; Hoem, 1997), dans les pays d'Afrique subsaharienne, les travaux soulignent une plus grande fragilité des unions des femmes instruites surtout dans les milieux urbains. A Lomé et à Dakar, par exemple, les femmes instruites ont plus de risque de divorcer que leurs con- sœurs non scolarisées (Antoine et Dial, 2005; Bocquier et Nanitelamio, 1991). La précarité économique des ménages, notamment le chômage du mari, est également un facteur de risque de divorce du couple. Socialement, dans le contexte africain, le mari a l'obligation d'assumer les charges du ménage, à défaut, l'union peut être fragilisée. Par contre, l'autonomie financière des femmes augmente le risque de divorce (Dial, 2007 ; Takyi et Broughton, 2006).

Toutefois, comme nous l'avons déjà souligné, les données du moment parfois utilisées sont inappropriées à l'étude du divorce. L'analyse transversale ne rend pas compte de la dynamique matrimoniale. Étant donné que le risque de rompre une union dépend du temps d'exposition au risque, il est nécessaire de tenir compte de la durée de l'union dans les analyses, c'est ce que permet l'analyse des biographies. Cette méthode permet d'inclure des variables explicatives qui évoluent dans le temps, et de mieux tenir compte des changements de situation intervenus dans la vie de l'individu, afin d'identifier les facteurs de risque de divorce. Son utilisation nécessite des données adaptées provenant idéalement d'enquêtes longitudinales, comme c'est le cas dans cette étude.

En fonction de la littérature existante, nous faisons l'hypothèse que le risque de divorce des couples est associé à la fois à des facteurs démographiques et socioéconomiques. De façon spécifique, nous formulons deux hypothèses. Premièrement, nous supposons que, dans le contexte du Burkina Faso, où les familles attribuent une grande importance à la procréation, l'infécondité du couple est un facteur de risque de divorce. Deuxièmement, nous présumons que les femmes vivant dans des localités développées (urbaines et rurales), qui sont davantage en contact avec d'autres modes de vie liés à la modernisation, ont un plus grand risque de divorce que les autres.

\section{Méthodologie \\ Données}

Nous avons utilisé les données de l'Enquête Migrations, Insertion Urbaine et Environnement au Burkina Faso (EMIUB), réalisée en 2000, conjointement par l'Institut Supérieur des Sciences de la Population (ISSP, ex UERD) de l'université de Ouagadougou, le département de démographie de l'université de Montréal et le CERPOD du Mali. Bien que le principal objectif fût la migration et l'insertion urbaine, des données ont été collectées sur l'histoire matrimo- 
niale, les activités économiques des individus et l'histoire génésique des femmes. L'échantillon des répondants a été choisi de manière aléatoire à partir de l'enquête ménage. Pour chaque individu âgé de 15 à 64 ans, la section sur l'histoire matrimoniale a recueilli les informations suivantes par union : date de cohabitation, âge au début de la cohabitation ainsi que l'ethnie et l'activité du conjoint. Pour les unions rompues, la date de fin de l'union et le motif de la rupture (divorce, séparation, veuvage) ont également été enregistrés. Environ 8647 biographies individuelles ont été reconstituées dont 4685 biographies de femmes (Poirier et al., 200I).

Ces données ont l'avantage de permettre la combinaison des histoires matrimoniale, génésique, résidentielle et professionnelle des femmes. Cependant, comme toute enquête rétrospective, il y a un effet de sélection de l'échantillon car seules les femmes ayant survécu au décès et aux migrations externes sont enquêtées. Les enquêtes rétrospectives peuvent aussi produire des omissions volontaires et involontaires (Beckett et al., 200I). Les informations recueillies faisant appel à la mémoire des enquêtées, certaines unions lointaines de très courte durée pourraient être omises. Cependant, l'utilisation de la fiche "Âge-événement » lors de l'enquête a permis d'améliorer la qualité des données (Antoine, 2002).

Les données individuelles sont complétées par celles de l'enquête biographique communautaire (2002) qui a collecté des informations rétrospectives sur les caractéristiques du contexte local susceptibles d'influencer les comportements démographiques des populations. Ces données ayant été collectées pour être liées aux données individuelles, il aurait été souhaitable d'enquêter dans toutes les localités citées dans les histoires migratoires des individus. Mais pour des raisons financières et logistiques, ces localités se répartissaient sur l'ensemble du territoire du pays, ces données ont été collectées seulement dans les localités où au moins trois épisodes de résidence ont été enregistrés lors de l'enquête biographique individuelle.

Environ 600 biographies de localités de résidence ont ainsi été reconstituées à l'aide d'un questionnaire communautaire administré simultanément à plusieurs informateurs, regroupant dans la plupart des cas le chef du village et/où le délégué du village (représentant administratif) ainsi que d'autres représentants (jeunes, femmes, groupes ethniques). Pour chaque localité, des informations ont été collectées de façon rétrospective jusqu'en 1960 sur l'histoire administrative, les infrastructures scolaires et sani- taires, l'équipement collectif et les activités agricoles (Schoumaker et al., 2006). Ces données fournissent ainsi les caractéristiques du contexte dans lequel vivent les individus à chaque période au cours de leur vie. Pour permettre de coupler les données communautaires avec les données individuelles, des imputations (par une méthode d'imputation aléatoire hot-deck) ont été faites pour tous les villages dans lesquels ont vécu des individus, mais qui n'ont pas été compris dans l'enquête communautaire (voir Beauchemin et Schoumaker, 2005 pour plus de détails). Toutes les villes (localités de plus de 10000 habitants) concernées ont été enquêtées, il n'y a donc pas eu de données manquantes pour les villes. L'imputation des valeurs manquantes pour les données communautaires pourrait introduire des biais dans l'analyse, mais les critères de choix des villages enquêtés pour remplacer ceux qui n'ont pas été concernés ont permis de réduire ces biais. En effet, deux principaux critères ont été utilisés pour le choix des villages de substitution : la province dans laquelle est situé le village concerné (le Burkina Faso compte 45 provinces) et la taille de la population (moins de 5000 ou 5000 à 10000 habitants).

L'échantillon d'analyse regroupe 387I femmes âgées de 15 à 64 ans ayant été en union. Dans l'enquête, le terme union s'applique à l'ensemble des femmes qui se sont déclarées mariées ou vivant maritalement avec quelqu'un à un moment donné de leur vie. L'âge moyen au premier mariage des femmes est de 18 ans. On note des différences selon l'ethnie, il est par exemple de 16,5 ans chez les Peul, 17 ans chez les Gourmantché et 18,4 ans chez les Mossi. Selon la religion, l'âge moyen au premier mariage est de 17,8 ans pour les musulmanes et de 18 ans pour les animistes et les chrétiennes.

\section{Variables d'analyse}

La variable dépendante de l'analyse est le risque instantané de connaître la rupture de sa première union par divorce. Le temps d'analyse est la durée de l'union. II est calculé par différence entre la date de fin de la première union ou la date de l'enquête pour les femmes qui sont toujours dans leur première union (observations tronquées) et la date de début de l'union (début de la cohabitation).

Les principales variables explicatives sont des variables de fécondité, précisément le nombre d'enfants survivants et la composition par sexe des enfants de la femme. Le nombre d'enfants survivants par femme varie de zéro à cinq enfants et plus. Mais, au-delà du nombre, est ce que le sexe des enfants a un effet sur le risque de divorce? Plus spécifique- 
ment, est ce que le fait de ne pas avoir de garçon, augmente le risque de séparation du couple? Pour cela, nous avons distingué trois catégories de femmes: celles qui ont uniquement des garçons, celles qui ont uniquement des filles et celles qui ont des enfants des deux sexes. Afin d'observer l'effet du contexte local sur le risque de divorce, nous avons construit un indicateur de niveau de développement en utilisant les variables communautaires suivantes : écoles primaire et secondaire, service de santé, téléphone, électricité, adduction d'eau potable, marché, route, utilisation de la charrue et du tracteur et activités de loisir. Le choix de ces variables s'inspire du rapport de la commission mondial sur l'environnement et le développement de I'ONU, appelé "Rapport Brundland", paru en avril 1987 (source : http://www.mediaterre.org). À l'aide de la méthode d'analyse en composante principale, nous avons créé et retenu le premier facteur dont la valeur explicative du niveau de développement est d'environ $77,4 \%$. Les localités ont ensuite ont été divisées en deux groupes à l'aide de cet indice pour créer un indicateur de développement : localités développées et localités non développées. En tenant compte du fait qu'au Burkina Faso le niveau de développement des localités est fortement lié au type de résidence, les villes étant généralement mieux équipées que les villages, nous avons ensuite croisé cet indicateur avec le milieu de résidence pour constituer trois catégories : rural peu développé, rural développé et urbain.

L'analyse inclut également d'autres variables explicatives: la cohorte de mariage, le niveau d'instruction (plus haut niveau atteint), l'âge au premier mariage, le milieu de résidence, le milieu de socialisation (lieu de résidence à l'âge de 6 ans), la religion, l'ethnie et le statut d'activité de la femme, l'activité du conjoint (de la première union) et la composition ethnique du couple (même ethnie ou ethnies différentes).

Les variables indépendantes nombre d'enfants survivants, composition par sexe des enfants, milieu de résidence, statut dans l'activité de la femme et l'indicateur de niveau de développement changent au cours du temps. Les autres variables ont des valeurs constantes. Bien que le niveau d'instruction puisse évoluer avec le temps, nous l'avons considéré invariable compte tenu de la faible proportion de femmes scolarisées au Burkina Faso, et du fait qu'au moment du mariage, dans la plupart des cas, la femme a achevé (ou interrompu définitivement) sa scolarité. Au Burkina Faso on dénombre plus de 60 ethnies regroupées en 12 groupes. Pour des raisons d'effectifs, nous avons retenu les six premiers groupes (le groupe Mossi rassemble près de la moitié de la population), les autres sont réunis dans la modalité "autres ethnies". Cependant, les variables différence d'âges entre conjoints (l'âge du conjoint a été renseigné par la femme) et polygamie souvent utilisées dans les études sur le divorce ne sont pas incluses à cause de la mauvaise qualité des données. De plus, les données ne fournissent aucune information concernant le choix du conjoint.

Sur les 3871 femmes de l'échantillon d'analyse, 356 ont rompu leur première union par divorce ou séparation et $37 \mathrm{I}$ n'ont pas eu d'enfants survivants au moment de leur sortie d'observation. Le tableau I présente les caractéristiques de la population étudiée et indique les différentes catégories des variables explicatives. Pour des raisons pratiques, nous avons rassemblé les femmes en trois cohortes de mariage : la plus ancienne composée de femmes mariées avant 1975 représente environ $25 \%$ de l'échantillon ; la cohorte intermédiaire (1975-1989) et la jeune cohorte (1990-2000) représentent respectivement $36 \%$ et $39 \%$ de l'échantillon. La classification du milieu de résidence et de socialisation tient compte du processus d'urbanisation au Burkina Faso (Beauchemin et al., 2002). La majorité des enquêtées $(89 \%)$ a été socialisée en milieu rural. Les données confirment aussi la faible scolarisation des femmes $(88 \%$ sont sans instruction) et la précocité du premier mariage au Burkina Faso. Plus de $\mathbf{9 0 \%}$ des femmes se sont mariées avant l'âge de 20 ans. La pratique de l'endogamie ethnique est répandue puisque 9 femmes sur 10 sont mariées à un homme du même groupe ethnique. 


\begin{tabular}{|c|c|c|c|c|}
\hline Variables & $\begin{array}{l}\text { Effectifs } \\
\text { non pond. }\end{array}$ & $\begin{array}{l}\text { Effectifs } \\
\text { pondérés }\end{array}$ & $\begin{array}{l}\text { Proportion } \\
\text { (\%) }\end{array}$ & $\begin{array}{l}\text { Nb. années } \\
\text { vécues }\end{array}$ \\
\hline \multicolumn{5}{|l|}{ Nb. d'enfants survivants } \\
\hline 0 enfant & 359 & 370,5 & 9,6 & II 835,7 \\
\hline I enfant & 655 & 745,3 & 19,3 & 12851,6 \\
\hline 2 enfants & 594 & 577,3 & 14,9 & 10069,7 \\
\hline 3 enfants & 585 & 555,7 & 14,4 & 9011,4 \\
\hline 4 enfants & 485 & 442,3 & 11,4 & 7759,0 \\
\hline $5+$ enfants & 1193 & I 179,9 & 30,5 & 14800,6 \\
\hline \multicolumn{5}{|l|}{ Sexe des enfants $(v)$} \\
\hline Aucun enfant & 375 & 370,5 & 9,6 & II 835,7 \\
\hline Uniquement garçons & 636 & 698,7 & 18,1 & 12220,0 \\
\hline Uniquement filles & 590 & 645,0 & 16,7 & 10318,9 \\
\hline Garçons et filles & 2270 & 2156,8 & 55,7 & 31953,4 \\
\hline \multicolumn{5}{|l|}{ Génération de mariage } \\
\hline Avant 1975 & 987 & 977,5 & 25,3 & 24893,1 \\
\hline $1975-1989$ & 1620 & | 398,I & 36,1 & 27349,1 \\
\hline $1990-2000$ & I 264 & I 495,4 & 38,6 & 14085,8 \\
\hline \multicolumn{5}{|l|}{ Age au ler mariage } \\
\hline moins de 15 ans & 620 & 569,8 & 14,7 & 10000,2 \\
\hline $15-17$ ans & I 747 & 1993,8 & 51,5 & 35108,8 \\
\hline $18-20$ ans & 1015 & 969,7 & 25,1 & 15918,8 \\
\hline plus de 20 ans & 489 & 337,7 & 8,7 & 5300,1 \\
\hline \multicolumn{5}{|l|}{ Niveau d'instruction } \\
\hline Aucune & 3000 & 3410,3 & 88,1 & $6|4| 1,7$ \\
\hline Primaire & 549 & 348,4 & 9,0 & 3805,2 \\
\hline Secondaire ou plus & 322 & 112,3 & 2,9 & I III,I \\
\hline \multicolumn{5}{|l|}{ Milieu de résidence $^{(v)}$} \\
\hline Rural & 2050 & 3035,7 & 78,4 & 53041,9 \\
\hline Urbain & 1776 & 786,2 & 20,3 & 9239,8 \\
\hline Étranger & 45 & 49,1 & $\mathrm{I}, 3$ & 4046,3 \\
\hline \multicolumn{5}{|l|}{ Milieu de socialisation } \\
\hline Rural & 2825 & 3437,2 & 88,8 & 60508,1 \\
\hline Villes moyennes & 466 & 169,8 & 4,4 & 2744,2 \\
\hline Grandes villes & 269 & 85,3 & 2,2 & 643,7 \\
\hline Étranger & 311 & 178,7 & 4,6 & 2431,9 \\
\hline \multicolumn{5}{|l|}{ Religion } \\
\hline Animiste & 680 & I 068,7 & 27,6 & 19474,8 \\
\hline Musulman & 2337 & $206 \mathrm{I}, 5$ & 53,3 & 34245,1 \\
\hline Chrétien & 844 & 732,2 & 18,9 & 12442,4 \\
\hline Autres & 10 & 8,6 & 0,2 & 165,7 \\
\hline \multicolumn{5}{|l|}{ Ethnie } \\
\hline Mossi & 1599 & I 885,4 & 48,7 & 32340,7 \\
\hline Gourmantché & 335 & $282, I$ & 7,3 & 4445,0 \\
\hline Peul & 325 & 287,2 & 7,4 & 5027,3 \\
\hline Gourounsi & 136 & 225,3 & 5,8 & 3750,6 \\
\hline Bobo-Bwa & 206 & 185,9 & 4,8 & 3227,2 \\
\hline Lobi-Dagari & 210 & 279,8 & 7,2 & 4870,1 \\
\hline Autres ethnies & 1060 & 725,2 & 18,7 & 12667,2 \\
\hline \multicolumn{5}{|l|}{ Ethnie du couple } \\
\hline Même ethnie & 3365 & 3595,0 & 92,9 & 61977,5 \\
\hline Ethnies différentes & 506 & 276,0 & 7,1 & 4350,5 \\
\hline \multicolumn{5}{|l|}{ Statut dans l'activité } \\
\hline Indépendant / employeur & 2804 & 3244,1 & 83,8 & 59403,1 \\
\hline Salariée & 788 & 265,2 & 6,9 & $348 \mathrm{I}, \mathrm{I}$ \\
\hline Apprentie / aide familiale & 199 & 323,4 & 8,4 & 2525,1 \\
\hline Autre & 80 & 38,3 & $\mathrm{I}, 0$ & 918,7 \\
\hline \multicolumn{5}{|l|}{ Activité du conjoint } \\
\hline Agriculture / Élevage / & 2279 & & & \\
\hline Pêche & & 3249,34 & 83,94 & $5766 \mathrm{I}, 4$ \\
\hline
\end{tabular}




\begin{tabular}{|c|c|c|c|c|}
\hline Cadre public / privé & 392 & $|4|, 8$ & 3,7 & I 748,9 \\
\hline Autres professions & 1134 & 447,6 & 11,6 & 6073,9 \\
\hline Inactifs & 66 & 32,3 & 0,8 & 843,8 \\
\hline $\begin{array}{l}\text { Indice de développe- } \\
\text { ent }\end{array}$ & & & & \\
\hline Rural peu développé & 2442 & I 788,40 & 46,2 & 37598,3 \\
\hline Rural développé & | $04 \mid$ & I 307,24 & 33,77 & 23251,3 \\
\hline Urbain & 388 & 775,36 & 20,03 & 5478,5 \\
\hline Total & 3871 & 3871 & 100 & 66328 \\
\hline
\end{tabular}

Source : Enquête "Migrations, Insertion Urbaine et Environnement au Burkina Faso" (EMUB, 2000).

(v) : Variables dont la valeur peut changer au cours du temps (répartition au moment de la sortie d'observation).

\section{Méthode d'analyse}

Nous avons utilisé la méthode d'analyse de survie, précisément, la méthode de l'estimateur de Kaplan Meier et la régression semi-paramétrique à risques proportionnels de Cox (Cleves et al., 2004; Courgeau et Lelièvre, 1989 ; Allison, 1984). Cette méthode permet d'étudier la survenue d'un évènement au cours du temps. Elle est également adaptée pour étudier des évènements vécus par une partie de la population étudiée, comme c'est le cas du divorce. Le divorce et le veuvage étant des évènements concurrents (une femme qui connaît le veuvage suite au décès de son conjoint ne peut plus connaître le divorce de cette union, et inversement), pour chaque union d'un individu, le veuvage est considéré ici comme une troncature.

Les estimés de Kaplan-Meier ont servi à construire les graphiques correspondant à la fonction de survie de la population étudiée. Mais cette méthode considère la population homogène et la distribution du risque est estimée pour l'ensemble de la population étudiée sans tenir compte des effets des caractéristiques individuelles. Elle ne permet donc pas de mesurer simultanément l'effet de plusieurs variables indépendantes. En revanche le modèle de Cox, qui est une méthode d'analyse explicative, permet de tenir compte de l'hétérogénéité des populations. L'avantage du modèle de Cox est de permettre la prise en compte du temps dans l'analyse du divorce et de tenir compte des variables changeant dans le temps. Mais son utilisation repose sur l'hypothèse de proportionnalité des risques, ce qui constitue une contrainte majeure. Cependant, cette hypothèse a été testée et elle est quasiment vérifiée au moins pour la principale variable indépendante (nombre d'enfants survivants).

\section{Résultats}

\section{Résultats descriptifs}

Le graphique I représente les courbes de survie au divorce des femmes à partir des valeurs estimées de Kaplan-Meier. Ces courbes indiquent la proportion de femmes encore en union à chaque durée de leur premier mariage. Les résultats montrent qu'au niveau national environ une femme sur 10 rompt sa première union par divorce au cours des 30 premières années de mariage. En milieu urbain cette proportion est atteinte après 20 ans d'union. Les différences sont significatives au seuil de $1 \% \circ$ selon le test log-rank. Toutefois, le risque de divorce varie selon la durée de l'union. II se situe à environ I,7 pour mille au début de l'union et augmente pendant les quatre premières années de mariage pour atteindre 2 pour mille, puis il diminue progressivement avec chaque année additionnelle de mariage.

L'évolution des courbes par cohorte de mariage montre des différences significatives (au seuil de I\%o d'après le test log-rank) entre générations de mariage. Le divorce apparaît de plus en plus rapide au sein de la jeune cohorte (1990-2000). Au sein de celle-ci environ $11 \%$ des unions sont rompues après 10 ans de mariage alors que cette proportion est respectivement de $2 \%$ et $4 \%$ pour les femmes mariées avant 1975 et celles mariées entre 1975 et 1989 pour la même durée d'union.

Cependant, l'affirmation selon laquelle les ruptures d'unions sont fréquentes dans les pays d'Afrique de l'Ouest (Lesthaeghe et al., 1989) n'est pas confirmée au Burkina Faso. Dans l'ensemble, le divorce est peu fréquent comparativement à d'autres pays. Par exemple, au cours des 30 premiers ans de mariage, la proportion des premiers mariages féminins rompus par divorce atteint 38\% au Togo, 35\% au Ghana, 29\% en Mauritanie (Tabutin et Schoumaker, 2004) et 15\% au Nigéria (IsiugoAbanihé, 1998), alors qu'elle est de 10\% au Burkina Faso. Le divorce est encore plus fréquent dans cer- 
tains pays d'Afrique de l'Est. II touche, par exemple, $45 \%$ des premières unions en Éthiopie, après 30 ans de mariage, (Tilson et Larsen, 2000), et $65 \%$ après
25 ans de mariage en milieu rural au Malawi (Reniers, 2003).

Graphique I : Probabilité pour les femmes de ne pas divorcer au cours de leur première union (valeurs estimées de Kaplan-Meier)
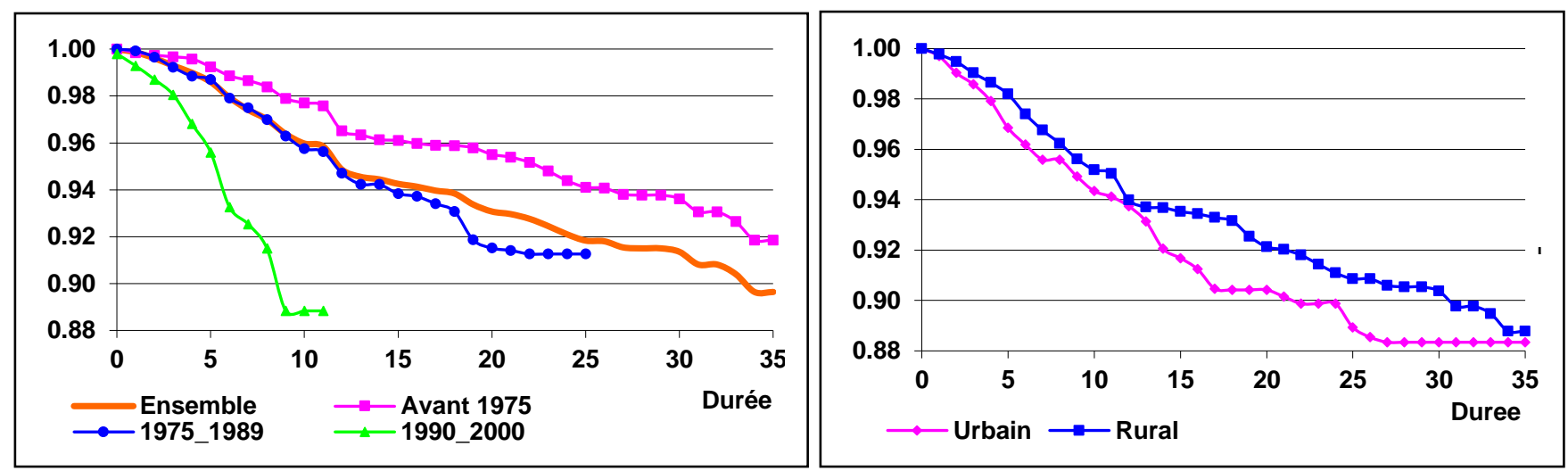

Source : Enquête "Migrations, Insertion Urbaine et Environnement au Burkina Faso" (EMUB, 2000)

\section{Résultats de l'analyse multivariée}

Le tableau 2 présente les résultats de deux modèles de régression de Cox concernant l'effet des variables indépendantes sur le risque de divorce de la première union des femmes. Le premier modèle évalue l'effet simultané des différentes variables individuelles sur le risque de rompre sa première union. Le second modèle prend en compte l'effet du niveau de développement de la localité de résidence. Les chiffres représentés dans le tableau sont les risques relatifs (odds ratio en anglais) de survenue du divorce pour chaque groupe par rapport à la catégorie de référence (celle entre parenthèse). $\mathrm{Si}$ le risque relatif est égale à $\mathrm{I}$, cela signifie une absence de différence de risque entre le groupe de référence et le groupe considéré, s'il est supérieur à I le risque de divorce est plus élevé pour le groupe considéré, c'est le contraire lorsque le risque relatif est inférieur à $\mathrm{I}$.

Dans ces modèles, la catégorie de femmes sans enfant a été scindée en deux sous-groupes en fonction de la durée de l'union : pendant les deux premières années d'union et après deux ans d'union. Nous supposons que l'effet négatif de l'infécondité augmente avec la durée de l'union, et qu'il est plus important après deux ans de mariage. En revanche, le fait pour un couple de ne pas avoir d'enfant pendant les deux premières années de l'union, ce qui correspond à l'intervalle protogénésique, peut-être moins aggravant. La limite de deux ans a été choisie en tenant compte de la différence entre l'âge médian au premier mariage (I 7,7 ans) et de l'âge médian à la première naissance (19,6 ans) des femmes au Burkina Faso (INSD et Macro international, 2003).

\section{L'infécondité, un facteur de divorce}

Les résultats montrent le rôle des enfants dans la stabilité des unions conjugales au Burkina Faso comme dans beaucoup de pays d'Afrique. Le risque de divorce est très associé au nombre d'enfants survivants de la femme. Les femmes sans enfants et celles qui ont un seul enfant présentent des risques plus élevés que celles qui ont 4 enfants. Comme nous l'avions présumé, pour les femmes sans enfants, le risque devient significativement plus important au-delà de deux ans de mariage, notamment lorsqu'on considère les variables individuelles (modèle I). En revanche, les femmes qui ont au moins 5 enfants survivants ont $61 \%$ moins de risque de divorce que celles qui ont quatre enfants. Ces résultats pourraient s'expliquer par la place de l'enfant au sein de la famille au Burkina Faso et dans la plupart des sociétés africaines.

Par contre, il semble que même si la femme, et particulièrement l'homme, souhaite incontestablement avoir des garçons pour assurer sa succession, le risque de divorce n'est pas associée au sexe de l'enfant. Ce résultat pourrait s'expliquer de différentes manières. Même si les garçons sont très désirés, les filles sont aussi valorisées. Elles sont par exemple importantes dans les échanges matrimoniaux, et elles constituent une importante aide pour les mères dans les travaux ménagers. La pratique de la polygamie permet également à l'homme 
d'atteindre son objectif d'avoir des garçons avec une autre épouse sans divorcer de la femme qui ne lui a fait que des filles.

\section{Effet du niveau de développement}

Comme présumé, les femmes vivant dans les localités urbaines et rurales développées sont associées à un plus grand risque de rupture d'union que celles du milieu rural peu développé. Elles ont 1,33 fois plus de risque de divorce que ces dernières, et les différences sont significatives. Les moyens de communication (route, téléphone) et le désenclavement des localités pourraient en partie expliquer ce résultat. Au Burkina Faso, chaque groupe social est solidaire des alliances matrimoniales de ses membres. Le remariage d'une femme divorcée avec un homme du même groupe ou d'un groupe allié est habituellement désapprouvé. Aussi, le remariage de la femme s'accompagne presque toujours de son installation dans un autre village. La perspective de se remarier, en cas de divorce, augmente alors avec l'existence de moyens de communications, et les marchés (permanents ou périodiques) sont d'excellents lieux de rencontre d'un futur époux. L'indicateur du niveau de développement introduit des différences de risque de ruptures d'unions conjugales même entre les localités rurales selon leur niveau d'équipement.

\section{Autres facteurs associés au risque de divorce}

Plusieurs autres facteurs sont également associés au risque de divorce. Les résultats de l'effet de l'âge au premier mariage des femmes vont dans le sens de la littérature sur l'Afrique. Comme observé dans d'autres travaux, les femmes mariées avant l'âge de 20 ans sont associées à un risque plus grand de rompre leur première union que les autres. Par exemple, les femmes qui se sont mariées avant l'âge de 18 ans ont 2,5 fois plus de risque de divorce que celles qui se sont mariées à l'âge de 20 ans et plus.

La variable cohorte de mariage, qui permet d'observer l'effet du contexte politique, économique et social du pays sur le risque de divorce, indique des différences significatives. Les femmes mariées dans les périodes récentes (1990-2000 et 19751989) ont un risque de divorce plus grand que celles de la plus vieille cohorte. Les difficultés économiques des dernières décennies, en entrainant la détérioration des conditions de vie des ménages pourraient aussi affaiblir la cohésion des couples et augmenter leur risque de divorce. Par ailleurs, les femmes socialisées en ville sont plus à risque que les autres. Par exemple, les femmes socialisées dans les grandes villes ont 2,4 fois plus de risque de divorce que celles socialisées en milieu rural.

Selon la religion, les femmes chrétiennes présentent un risque de divorce plus grand que les musulmanes. Le même constat a été fait au Nigéria (Isiugo-Abanihé, 1998) et au Ghana (Takyi, 200I). Par contre, à Dakar, où le christianisme est peu répandu, les femmes chrétiennes présentent des unions plus stables que les musulmanes (Bocquier et Nanitelamio, 1991). Toutefois, cet effet de la religion sur l'instabilité des unions est encore mal expliqué. Le mariage est une institution sociale reconnue et incitée par toutes les religions. Pour l'église chrétienne, le mariage crée un lien sacré entre les époux pour toute la vie. Au Burkina Faso, la plupart des églises accompagnent les futurs mariés dans leurs préparatifs au mariage par des enseignements, et certaines offrent des structures de conciliation des conjoints (conseiller conjugal) en cas de discorde. La religion musulmane déclare que le contrat conclu entre époux lors du mariage a pour objectif de durer à l'infini, et n'accepte le divorce que comme dernier recours. Chez les animistes, même si le mariage n'est pas formellement considéré indissoluble, le contrôle familial et social veille à la stabilité des unions. Par conséquent, l'explication de ces différences mérite des investigations plus spécifiques.

On note aussi des différences selon l'appartenance ethnique. Les femmes Peul, Lobi-Dagari, Gourounsi et Bobo-Bwa présentent un risque de divorce plus grand que les femmes Mossi. Le risque élevé de divorce chez les Peuls a déjà été observé dans plusieurs autres pays d'Afrique de l'Ouest, notamment au Sénégal (Pison, 1988; Antoine et Djiré, 1998) et au Togo (Thiriat, 1998, Antoine et Dial, 2005). Ces différences pourraient s'expliquer en partie par le type d'organisation sociopolitique de chaque groupe, les normes sociales matrimoniales varient selon le groupe ethnique. Par exemple, chez les Mossi le mariage entre personnes apparentées est prohibé, alors que chez les Peul, ce type d'union est permis, et le mariage entre cousins est encouragé. Lors du mariage, la femme Peul reçoit en dot un capital de bétail de la part de sa bellefamille, et afin de conserver les richesses de la famille, les parents encouragent le mariage consanguin (Dupire, 1970). Ainsi, si le premier objectif visé dans les alliances matrimoniales est davantage la sauvegarde des ressources de la famille que l'intérêt de la jeune fille, lorsque les conjoints sont insatisfaits de leur union, le divorce peut survenir. 
Par contre, contrairement à ce qu'on présumait, l'exogamie ethnique n'est pas un facteur de risque de divorce. Au contraire, les unions interethniques semblent plus stables que les mariages intraethniques. Toutefois, l'exogamie ethnique a été mentionnée comme facteur associé au risque de divorce dans des entretiens qualitatifs réalisés au Burkina Faso (Thiombiano, 2009).

La situation économique du ménage mesurée à l'aide du statut d'activité de la femme et de l'activité du conjoint (celui de sa première union) est aussi associée au risque de divorce. Les femmes salariées ayant une autonomie financière et celles dont les maris sont salariés (cadres du public ou privé) présentent un grand risque de divorce. Les femmes salariées ont 1,9 fois plus de risque de divorce que les travailleuses indépendantes (elles exercent surtout dans le secteur informel); tandis que les femmes dont les conjoints sont salariés ont 2,7 fois plus de risque de divorce que celles dont les conjoints sont agriculteurs ou éleveurs. Par contre les femmes apprenties et les aides familiales, qui n'ont pas de revenus personnels, ont un risque plus faible que les travailleuses indépendantes. 
Tableau 2 : Facteurs associés au divorce de la première union des femmes au Burkina Faso : résultats des régressions de Cox

\begin{tabular}{|c|c|c|}
\hline Variables indépendantes & Modèle I & Modèle 2 \\
\hline \multicolumn{3}{|c|}{ Nb. d'enfants survivants (v) (4 enfants) } \\
\hline 0 enfant avant 2 ans d'union & 5,18 **** & 4,97 **** \\
\hline 0 enfant après 2 ans d'union & 7,02 ***** & 4,44 ***** \\
\hline I enfant & 4,30 ***** & 3,21 ***** \\
\hline 2 enfants & 2,07 ***** & 1,67 **** \\
\hline 3 enfants & 1,24 & 1,08 \\
\hline 5 enfants et plus & 0,36 **** & 0,39 ***** \\
\hline \multicolumn{3}{|c|}{ Sexe des enfants ${ }^{(v)}$ (Uniquement garçons) } \\
\hline Uniquement des filles & 1,08 & 1,08 \\
\hline Garçons et filles & 0,91 & 0,91 \\
\hline \multicolumn{3}{|c|}{ Cohorte de mariage (Avant 1975) } \\
\hline $1975-1989$ & $|, 2|$ **** & 1,19 ***** \\
\hline $1990-2000$ & 1,28 ***** & 1,23 **⿻丷木 \\
\hline \multicolumn{3}{|c|}{ Age au premier mariage (20 ans et + ) } \\
\hline Moins de 15 ans & $2,62 * * * *$ & 2,53 **** \\
\hline 15 à 17 ans & 2,68 ***** & 2,58 ***** \\
\hline 18 à 20 & $1,60 * * * *$ & I,55 **** \\
\hline \multicolumn{3}{|l|}{ Instruction (Aucune) } \\
\hline Primaire & $\mathrm{I}, \mathrm{II} *$ & 1,09 \\
\hline Secondaire et supérieur & 1,16 & 1,15 \\
\hline \multicolumn{3}{|l|}{ Milieu de résidence ${ }^{(v)}$ (Rural) } \\
\hline Urbain & 1,30 & - \\
\hline \multicolumn{3}{|c|}{ Milieu de socialisation (Rural) } \\
\hline Villes moyennes & 1,53 ***** & 1,55 ***** \\
\hline Grandes villes & 2,18 ***** & 2,4 I ***** \\
\hline Étranger & 1,13 & $1,13 *$ \\
\hline \multicolumn{3}{|l|}{ Religion (Musulmanes) } \\
\hline Animistes & $0,88 * *$ & $0,94 *$ \\
\hline Chrétiennes & $1,30 * * * *$ & 1,33 **** \\
\hline Autres & 0,32 **** & 0,35 **** \\
\hline \multicolumn{3}{|l|}{ Ethnie (Mossi) } \\
\hline Gourmantché & 1,14 *** & 1,14 *** \\
\hline Peul & 2,12 **** & 2,09 **** \\
\hline Gourounsi & $|, 4|$ **** & 1,33 ***** \\
\hline Bobo-Bwa & 1,67 ***** & $\mathrm{I}, 52$ *⿻丷木**十 \\
\hline Lobi-Dagari & 2,49 **** & 2,55 **** \\
\hline Autres ethnies & $\mathrm{I}, 40$ **** & 1,39 **** \\
\hline \multicolumn{3}{|c|}{ Ethnie du couple (Même ethnie) } \\
\hline Ethnies différentes & $0,91 *$ & $0,90 *$ \\
\hline \multicolumn{3}{|c|}{ Statut dans l'activité(v)(Indépendante) } \\
\hline Salariée & 1,93 **** & $1,9 \mid$ **** \\
\hline Apprentie / Aide familiale & 0,84 **** & 0,84 *⿻丷木**十 \\
\hline Autres & 0,38 **** & 0,38 ***** \\
\hline \multicolumn{3}{|c|}{ Activité du conjoint (Agriculture / Élevage) } \\
\hline Cadre, public / privé & $2,79 * * * *$ & 2,73 ***** \\
\hline Autres professions & $1,48 * *$ & $\mathrm{I}, 45$ ***** \\
\hline Inactifs & 0,15 **** & 0,15 *⿻丷木**十 \\
\hline \multicolumn{3}{|c|}{ Indice de développement (Rural pas développé) } \\
\hline Rural développé & & 1,33 **** \\
\hline Urbain & & 1,33 ***** \\
\hline $\mathbf{N}$ & 3871 & $387 I$ \\
\hline
\end{tabular}

Niveaux de significativité : ${ }^{*} \mathrm{p}<0,05$; ${ }^{* *} \mathrm{p}<0,01$; ${ }^{* * *} \mathrm{p}<0,00 \mathrm{I}$; Catégorie de référence entre parenthèse. (v) : Variables dont la valeur peut changer au cours du temps (répartition au moment de la sortie d'observation). 


\section{Discussion}

Les résultats montrent que le divorce demeure relativement peu fréquent au Burkina Faso (environ $10 \%$ des premières unions féminines) comparativement à certains pays d'Afrique de l'Ouest. II reste perçu comme une transgression des bonnes mœurs, un échec et un déshonneur par les familles, et n'intervient généralement qu'après l'échec de conciliation du couple par les parents. Toutefois, on note que le divorce est plus fréquent au sein des jeunes générations de mariages qui éprouvent d'énormes difficultés d'accès à l'emploi avec la récession économique des dernières décennies (Calvès et Schoumaker, 2004). Plusieurs variables sont significativement associées à un risque plus élevé de divorce.

Ainsi, l'infécondité est un facteur important de risque de divorce. Le but explicite du mariage étant la procréation, une union inféconde a peu de chance de survivre. La pression sociale et familiale exercée sur le couple en cas d'infécondité peut être considérable, surtout lorsqu'il cohabite avec la famille élargie, ce qui peut augmenter le risque de séparation. En effet, sur le plan familial, avoir beaucoup d'enfants confère à la famille un prestige et un statut social important dans la communauté, particulièrement en milieu rural. De plus, sur le plan économique, les enfants constituent une source de main d'œuvre importante dans cette société d'agriculture traditionnelle. Dans ce contexte où le système de protection sociale moderne est inaccessible à tous, les enfants représentent aussi une sécurité sociale pour les parents (Maïga et Baya, 20I4).

Nous avions aussi supposé que le grand désir d'avoir des garçons dans ces sociétés pouvait influencer la stabilité des couples. Mais cette hypothèse n'a pas été confirmée. Ce résultat suggère que même si les garçons sont très désirés par les familles pour assurer la descendance, les filles sont aussi considérées. Elles sont par exemple nécessaires dans les alliances matrimoniales.

Par ailleurs, les facteurs de modernisation tels que l'urbanisation et le niveau de développement du lieu de résidence sont associés au risque de divorce. Les localités les plus développées offrent des lieux de rencontre et de pratiques qui pourraient favoriser les ruptures d'union. En effet, le remariage d'une femme divorcée avec un homme du même groupe ou d'un groupe allié étant réprouvé par les familles, le remariage de la femme se fait souvent dans un dans un autre village. Aussi, le niveau d'équipement comme les moyens de communications, en permettant les échanges entre localités et individus, augmentent les chances de remariage des femmes en cas de divorce.

Les femmes des jeunes générations de mariage présentent aussi des risques de divorce plus élevés. Traditionnellement, le rôle essentiel de la femme est d'être une épouse respectueuse et soumise, c'est le mari qui détient incontestablement l'autorité dans le ménage. Les jeunes femmes, étant en contact avec d'autres valeurs modernes, aspirent souvent à plus de considération et de rapports d'égalité dans leur ménage. Ces rôles aspirés entrent parfois en conflit avec les rôles habituellement dévolus à la femme par la société. Ces différences de perceptions entre hommes et femmes quant à leurs rôles et statuts dans le ménage, peuvent être source de tensions et de divorce.

Les facteurs culturels comme la socialisation en milieu urbain, la religion et l'appartenance ethnique sont aussi des facteurs significativement associés au risque de divorce. Les différences observées entre groupes ethniques pourraient s'expliquer par les disparités de la sanction sociale du divorce. Par exemple, d'après Pool (1972), les Bobo se scandalisent peu de la rupture d'un mariage, alors que le divorce est sévèrement réprouvé chez les Mossi. Le groupe mossi, plus hiérarchisé (en classe sociale, âge et genre) que les autres, pourrait exercer un contrôle social plus resserré sur les couples et contribuer à la stabilité des unions conjugales. Des différences de risque de divorce entre groupes ethniques ont aussi été soulignées dans des travaux antérieurs au Ghana, (Amoateng et Heaton, 1989), à Dakar, (Bocquier et Nanitelamio, 1991) et à Lomé (Antoine et Dial, 2005). Les différences observées entre les femmes selon la religion pourrait s'expliquer par le fait que certaines personnes se déclarent appartenir à une religion sans pour autant être pratiquantes, et par conséquent, ne pas nécessairement observer les préceptes de cette religion. Pour mieux cerner l'effet de la religion sur le comportement des individus, les informations sur la pratique sont nécessaires, mais elles sont rarement collectées. Les informations sur les préceptes de chaque religion par rapport au divorce et le niveau d'appropriation de ces préceptes par les individus sont aussi utiles pour comprendre ces différences.

\section{Conclusion}

Cette étude fournit d'importants résultats sur le niveau et les facteurs de risque de divorce au Burkina Faso. L'accroissement du risque de ruptures d'unions conjugales au sein des jeunes générations révèle une augmentation progressive du divorce et 
indique des changements de comportements matrimoniaux au sein de la population burkinabè. Plusieurs variables sont significativement associées à un risque plus élevé de divorce. Comme nous l'avions présumé, l'infécondité du couple est associée à un risque plus élevé de divorce, ce qui confirme l'importance de la reproduction dans les stratégies matrimoniales. De plus, comme observé ailleurs, le jeune âge au premier mariage, l'instruction et l'autonomie financière de la femme sont aussi positivement associé au risque de divorce.

Cependant, les résultats suggèrent que si pour l'instant le divorce s'inscrit en négatif dans les représentations sociales du Burkina Faso, il pourrait être l'expression d'un libre choix des femmes. En effet, plusieurs variables associées au risque de divorce sont aussi associées à un statut socioéconomique élevé de la femme. II s'agit notamment de la résidence et de la socialisation en milieu urbain et de l'autonomie financière de la femme. Malgré les limites des données communautaires, les analyses montrent que le niveau de développement du lieu de résidence introduit des différences entre les femmes. Ce résultat est remarquable même en milieu rural ; les femmes vivant dans des localités rurales développées sont associées à un plus grand risque de divorce que celles des villages peu développés. Toutefois, pour mieux explorer et comprendre l'effet de certains facteurs, comme les facteurs culturels, sur le risque de divorce, des études plus spécifiques sont nécessaires, et elles pourraient aussi apporter un éclairage à la question des rapports de genre et au statut de la femme dans les sociétés burkinabè.

\section{Bibliographie}

Allison, D. P., 1984. Event History Analysis: Regression for Longitudinal Event Data, Series Quantitative Applications in Social Sciences, New Delhi, Sage Publications, Number 046, 87p.

Amato, P. R. et Previti D., 2003. "People's reasons for Divorcing: Gender, Social Class, the Life Course, and Adjustment", Journal of Family Issues, 24 (5), p. 602-626.

Amoateng, A. Y. et Heaton T. B., 1989. "The sociodemographic correlates of the timing of divorce in Ghana", Journal of Comparative Family Studies, 20 (I), p. 79-96.

Andersson, G., 1997. "The Impact of Children Divorce Risks of Swedish Women", European Journal of Population, 13, p. 109-145.
Antoine, P., 2002. « Les complexités de la nuptialité : de la précocité des unions féminines à la polygamie masculine en Afrique ", in : Graziella Caselli, J. Vallin et G. Wunsch, éd., Démographie : analyse et synthèse II. Les déterminants de la fécondité, $\mathrm{Pa}$ ris, INED, p. 75-102.

Antoine, P. et Bocquier P., 1999. "L'enquête Insertion urbaine à Dakar et Pikine », in : Groupe de réflexion sur l'approche biographique (éd.), Biographie d'enquête. Bilan de 14 collectes biographiques, Paris, INED (collection Méthodes et savoirs), p. 177-201.

Antoine, P. et Dial F. B., 2005. « Mariage, divorce et remariage à Dakar et Lomé ", in : K. Vignikin et P. Vimard (dir.), Familles au Nord, Familles au Sud, Academia-Bruylant, Louvain-la-Neuve, p. 205232.

Antoine, P. et Djiré M., 1998. "Crise et évolution des comportements matrimoniaux à Dakar ", in : Gendreau F., éd., Crises, pauvreté et changements démographiques dans les pays du sud, Paris, AUPELF-UREF et Éditions ESTEM, p. 203-235.

Beauchemin, C. et Schoumaker B., 2005. "Migration to cities in Burkina Faso: Does the level of development in sending areas matter?", Word Development, 33 (7), p. II29-II 52.

Beauchemin, C.,EBeauchemin E. et Le Jeune G., 2002. Tabvilles BF :Rapport de présentation, $E M U B$, document technique d'analyse, $n^{\circ} 2002-I$, non publié.

Becker, S. G., 1974. "A theory of marriage", in: Economics of the family: Marriage, children, and human capital, Schultz T. W. (éd.), Chicago: University of Chicago Press, p. 299-344.

Becker, S. G., 198I. A Treatise on the Family, Cambridge: Harvard University Press, $288 \mathrm{p}$.

Becker, S. G., Landes E. M. et Michael R., 1977. "An Economic Analysis of Marital instability", Journal of Political Economy, 85 (6), p. I |4 I- I I 87.

Beckett, M., Davanzo J., Sastry N., Panis C. et Peterson C., 200I. "The Quality of Retrospective Data". The journal of human resources, 36 (3): 593-625.

Bocquier, P. et Nanitelamio J., 1991. «Les déterminants socio-économiques des changements matrimoniaux des femmes de Dakar ", in : UEPA, éd., Actes de la conférence "Femme, Famille et Population", Ouagadougou, 24-29 avril 1991,vol. I, communications sollicitées, Dakar, UEPA, p. 369386. 
Booth, A. et Edwards J. N., 1985. "Age at marriage and marital instability", Journal of Marriage and the Family, 47 (I), p. 67-75.

Bumpass, L., Martin T. C. et Sweet J. A., 199I. "The Impact of Family Background and Early Marital Factors on Marital Disruption", Journal of Family Issues, 12 (I), p. 22-42.

Burkina Faso, 1990, Code des personnes et de la famille, Ouagadougou, $224 \mathrm{p}$.

Calvès, A. et Schoumaker B., 2004. "Crise économique et évolution de l'emploi des jeunes citadins au Burkina Faso ", Étude de la population africaine, 19 (I), p. 35-58

Clarke, L. et Berrington A., 1999. "Sociodemographic Predictors if Divorce", in : High Divorce rates: the State of the Evidence on Reasons and Remedies, London, Report published by the Lord Chancellor's Department, vol. I (Papers I3) Research series $N^{\circ} 2,37 p$.

Cleves, A. M., Gould W. W. et Gutierrez R. G. 2004. An Introduction to Survival Analysis, Revised Edition, Texas, A Stata Press, $30 \mathrm{lp}$.

Courgeau, D. et Lelievre E., 1989. Analyse démographique des biographies, Paris, INED, 289 p.

Dial, F. B., 2007. "Le divorce, une source d'émancipation pour les femmes ? Une enquête à Dakar et Saint-Louis ", in : Locoh T. (dir.), Genre et sociétés en Afrique. Implication pour le développement, Paris, INED (Les cahiers de l'Ined $\mathrm{n}^{\circ}$ 160), p. 357-372.

Dupire, M., 1970. Organisation sociale des peuls: étude d'ethnographie comparée, Paris, Librairie Plon, $624 \mathrm{p}$.

Hertrich, V., 1996. Permanences et changements de l'Afrique rurale: dynamiques familiales chez les Bwa du Mali, Les Études du CEPED, $\mathrm{n}^{\circ}$ 14, Paris, CEPED, $548 \mathrm{p}$.

Hertrich, V. et Locoh T., 1999. Rapport de genre, formation et dissolution des unions dans les pays en développement, Liège, UIESP, 46 p. (Gender in population series).

Hoem, J. M., 1997. "Educational Gradients in Divorce Risks in Sweden in Recent Decades", Population Studies, 5 I (I), p. 19-27.

Institut national de la statistique et de la démographie et Macro International, 2003. Enquête Démographique et de Santé, Burkina Faso, 2003, Ouagadougou / Calverton, $455 \mathrm{p}$.

Isiugo-Abanihé, U. C., 1998. "Stability of marital union and fertility in Nigeria", Journal of Biological Science, 30: 33-4I.

Lesthaeghe, R., Kaufmann G. et Meekers D., 1989. « The Nuptiality Regime in Sub-saharian Africa ", in: Lesthaeghe R., éd., Reproduction and Social Organization in Sub-Saharan Africa, University of California Press, p. 238-237.

Levinger, G., 1965. "Marital Cohesiveness and Dissolution: An Integrative Review", Journal of Marriage and the Family, 27 (I), p. 19-28.

Locoh, T., 1995. Famillesafricaines, population et qualité de la vie, Les dossiers du CEPED, $\mathrm{n}^{\circ} 31$, Paris, CEPED, $48 \mathrm{p}$.

Locoh, T. et Mouvagha-Sow M., 2005. «Vers de nouveau modèles familiaux en Afrique de l'ouest", communication présentée au XXVème congrès international de la population(Tours 2005) séance s I I0I "La famille en Afrique", $28 \mathrm{p}$.

Maîga, A. et Baya B., 2014. "La coexistence des générations en milieu urbain au Burkina Faso : Entre logique de sélection et obligation de parenté», African Population Studies, 28 (2) Supplement July 20I4, p. I088-I I02.

Marcoux, R., 1997. "Nuptialité et maintien de la polygamie en milieu urbain au Mali", Cahiers québécois de démographie, 26 (2). p. 19|-2।4.

Mongeau, G., Neill G. et Le Bourdais C., 200I. «Effet de la précarité économique sur la formation d'une première union au Canada ", Cahier québécois de démographie, 30 (I), p. 3-29.

Mouvagha-Sow, M., 2003. Formation et ruptures des unions dans un contexte de pauvreté au Gabon, communication présentée à la quatrième conférence sur la population, Tunisie, 8-12 décembre 2003, 16 p.

Olusanya, P. O., 1970. "A note on some factors affecting the stability of marriage among the Yoruba of western Nigeria", Journal of Marriage and the Family, 32 (I), p. I50-I55.

Oppenheimer, V. K., 1994. "Women's rising employment and the future of the family in industrial societies", Population and development Review, 20 (2), p. 293-349.

Oppenheimer, V. K., 1997a. "Comment on, The divorce and separation in the United States, 1880-1990", Demography, 34 (4), p. 467-472.

Oppenheimer, V. K., 1997b. “Women's employment and the gain to marriage: The specialization and trading model", Annual Review of Sociology, 23: $431-453$

Pison, G., 1988. "Polygamie, fécondité et structures familiales ", in : Dominique Tabutin, éd., Population et sociétés en Afrique au sud du Sahara, Paris, Edition L'Harmattan, p. 249-278.

Poirier, J., Piché V., Le Jeune G., Dabiré B. et Wane H. R., 200I. "Projet d'étude des stratégies de reproduction des populations sahéliennes à partir 
de l'enquête "Dynamique migratoire, insertion urbaine et environnement au Burkina Faso" », Cahiers québécois de démographie, 30 (2), p. 289309.

Pool, J., 1972. "Conjugal patterns in Upper Volta », in: D. Ian Pool, Sidiki Coulibaly, Demographic transition and cultural continuity in the Sahel: Aspects of the social demography of Uper Volta, Ithaca / New York, Cornell University, p. 38-97.

Reniers, G., 2003. "Divorce and remarriage in rural Malawi", Demographic Research Special Collection: I (6), p. I75-206.

Ruggles, S., 1997a. "The rise of divorce and separation in the united States, 1880-1990", Demography, 34 (3), p. 455-466.

Ruggles, S., 1997b. "The reply to Oppenheimer and Preston”, Demography, 34 ( ${ }^{\circ}$ 4): 475-479.

Schoumaker, B., Dabiré H. B. et GnoumouThiombiano B., 2006. «Collecter des biographies contextuelles pour étudier les déterminants des comportements démographiques: l'expérience d'une enquête au Burkina Faso ", Population, 61 (I-2), p. 77-I06.

South, S., 1995. "Do you need to shop around? Age at marriage, spousal alternatives and marital dissolution", Journal of Family Issues, 16: 432-449.

Spitz, G., 1988. "Women's employment and family relations: a review", Journal of Marriage and the family, 50 (3), p. 595-618.

Tabutin, D. et Schoumaker B., 2004. "La démographie de l'Afrique au sud du Sahara des années 1950 aux années 2000, synthèse des changements et bilan statistique ", Population, 59 (3-4), p. 52I-622.
Takyi, B., 200I. "Marital instability in an African society: exploring the factors that influence divorce processes in Ghana", Sociological Focus, 34 (I), p. 77-96.

Takyi, K. B. et Broughton C., 2006. "Marital instability in Sub-Saharan Africa: Do women's autonomy and socioeconomic situation", Journal of Family and Economic Issues, 27 (I), p. II3-I3I.

Thiombiano, B,. 2009. Rupture des unions conjugales au Burkina Faso : causes et effets sur les femmes et leurs enfants, thèse de doctorat, Montréal, canada, $174 \mathrm{p}$.

Thiriat, M., 1998. Faire et défaire les liens du mariage : évolution des pratiques matrimoniales au Togo, Les Études du CEPED n 16, Paris, CEPED, $295 \mathrm{p}$.

Thornton, A. et Rodgers W., 1987. "The Influence of Individual and Historical Time on Marital Dissolution”, Demography, 24 (I), p. I-22.

Tilson, D. et Larsen U., 2000. "Divorce in Ethiopia: The impact of early marriage and childlessness", Journal of Biosocial Science, 32, p. 355-372.

Udry, J. R., 198I. "Marital alternatives and Marital Disruption", Journal of Marriage and the Family, 43 (4), p. 889-897.

White, L. K., 1990. "Determinants of divorce: A review of research in the eighties", Journal of Marriage and the Family, 52 (4), p. 904-912. 